\title{
Extraretinal and retinal amplitude and phase errors during Filehne illusion and path perception
}

\author{
TOM C. A. FREEMAN \\ University of Califormia, Berkeley, Califormia \\ and Cardiff University, Cardiff, Wales \\ MARTIN S. BANKS \\ University of Califormia, Berkeley, California \\ and \\ JAMES A. CROWELL \\ University of California, Berkeley, Califormia \\ and Califormia Institute of Technology, Pasadena, California
}

\begin{abstract}
Pursuit eye movements give rise to retinal motion. To judge stimulus motion relative to the head, the visual system must correct for the eye movement by using an extraretinal, eye-velocity signal. Such correction is important in a variety of motion estimation tasks including judgments of object motion relative to the head and judgments of self-motion direction from optic flow. The Filehne illusion (where a stationary object appears to move opposite to the pursuit) results from a mismatch between retinal and extraretinal speed estimates. A mismatch in timing could also exist. Speed and timing errors were investigated using sinusoidal pursuit eye movements. We describe a new illusion-the slalom illusionin which the perceived direction of self-motion oscillates left and right when the eyes move sinusoidally. A linear model is presented that determines the gain ratio and phase difference of extraretinal and retinal signals accompanying the Filehne and slalom illusions. The speed mismatch and timing differences were measured in the Filehne and self-motion situations using a motion-nulling procedure. Timing errors were very small for the Filehne and slalom illusions. However, the ratios of extraretinal to retinal gain were consistently less than 1 , so both illusions are the consequence of a mismatch between estimates of retinal and extraretinal speed. The relevance of the results for recovering the direction of self-motion during pursuit eye movements is discussed.
\end{abstract}

When we make a smooth eye movement to track a moving object, the visual system must take the eye's movement into account in order to estimate the object's velocity relative to the head. In principle, one can add the eye pursuit velocity $(\mathbf{P})$ to the observed retinal velocity $(\mathbf{R})$ to estimate the head-centric velocity of the object $(\mathbf{H})$. Of course, the visual system must also measure the retinal and eye pursuit velocities and those measurements may be subject to error. If we represent the errors as gains (Freeman \& Banks, 1998), the estimation of head-centric velocity is given by the following:

$$
\hat{\mathbf{H}}=r(\Omega) \mathbf{H}+\mathbf{P}[e-r(\Omega)],
$$

Some of these results appeared in preliminary form at the annual meeting of the Association for Research in Vision and Ophthalmology, Fort Lauderdale, Florida (Freeman, Crowell, \& Banks, 1996). We thank Cliff Schor and Jim Maxwell for help in the recording of eye movements and Karsten Weber and Payam Saisan for programming assistance. This research was supported by Grants AFOSR 93NL366 and NSF DBS9309820. T.C.A.F. is in the School of Optometry at the University of California; J.A.C. is in the Division of Biology at Cal Tech. Correspondence should be addressed to T. C. A. Freeman, Cardiff University, School of Psychology, P.O. Box 901, Cardiff CF10 3YG, Wales (e-mail: freemant@cardiff.ac.uk). where $e$ is the extraretinal gain factor relating actual to estimated eye speed and $r$ is the retinal gain, which varies with several stimulus parameters $(\Omega)$, including spatial frequency (Smith \& Edgar, 1990), dot density (Watamaniuk, Grzywacz, \& Yuille, 1993), and contrast (Thompson, 1982).

In this paper we consider how gaze rotations due to smooth eye movements affect perceived direction of selfmotion. When gaze rotation is zero, an observer translating along a straight path produces an expanding motion pattern in the retinal image. The retinal coordinates of the focus of expansion in this pattern correspond with the retinal coordinates of the direction of self-motion. Thus, when gaze direction is fixed relative to the direction of linear translation, the position of the focus of expansion can be used to recover the self-motion direction (Gibson, Olum, $\&$ Rosenblatt, 1955). When gaze direction varies relative to the path-for example, when the observer maintains fixation on an object to the side of the path-rotational flow is added to the retinal flow pattern and the focus is thereby eliminated. Nonetheless, human observers are able to recover the direction of self-motion during gaze rotations quite accurately (Crowell, Banks, Shenoy, \& Andersen, 1998; Royden, Banks, \& Crowell, 1992; Royden, 
Crowell, \& Banks, 1994; Warren \& Hannon, 1988, 1990). In other words, the visual system is able to compensate for the consequences of gaze rotation. We will refer to the means by which the visual system takes gaze rotation into account as gaze compensation.

There are a variety of ways in which gaze compensation could be accomplished. Two extremes are represented by extraretinal and retinal-image-based methods. The first method uses an extraretinal, eye-velocity signal (such as a copy of the efferent signal sent to the eye muscles) to estimate the gaze rotation and thereby compensate for the effects of the eye movement on the retinal motion pattern (Royden et al., 1992). The second method is accomplished from visual information alone (e.g., Heeger \& Jepson, 1992; Longuet-Higgins \& Prazdny, 1980; Perrone \& Stone, 1994). More recent work has shown how these two approaches could be combined (Beintema \& van den Berg, 1998).

Researchers have asked recently whether, in practice, extraretinal signals are involved in path perception during pursuit eye movements (e.g., Royden et al., 1992; Warren \& Hannon, 1988). In that research, path perception was compared in two conditions that yield the same retinalimage motion: a real eye-rotation condition in which the eye rotates, and a simulated rotation condition in which the eye is stationary and rotational flow is added to the display. Because the only difference between the two conditions is whether a pursuit eye movement is made or not, differences in the resulting path percepts reflect the contribution of an extraretinal, eye-velocity signal to the gaze compensation process in path perception. Some research has shown that the percepts differ significantly between the real and simulated rotation conditions. In the simulated rotation condition, observers have reported that they are moving on a curved path rather than the depicted linear path (van den Berg, 1996; Royden et al., 1992; Royden et al., 1994). Other studies have shown that human observers can, under some conditions, estimate the self-motion path reasonably accurately in the simulated rotation condition (van den Berg, 1996).

Estimation errors actually occur in both the real and simulated rotation conditions. For example, consider the data from Banks and colleagues (Banks, Ehrlich, Backus, \& Crowell, 1996; Ehrlich, Beck, Crowell, Freeman, \& Banks, 1998; Royden et al., 1992; Royden et al., 1994). In all of their experiments, path percepts were more accurate in the real than in the simulated eye-rotation condition, but small errors did occur in the real rotation condition. In particular, observers' responses were displaced slightly, but consistently, in the direction of the eye's rotation in the real eye-rotation condition. Freeman (1999) examined the path perception errors that accompany real eye pursuit in greater detail. He showed that nonzero simulated eye rotation must be introduced into displays depicting straight paths in order to null perceived path curvature. In this paper, we present a situation in which errors in gaze compensation are more salient. Observers viewed displays depicting forward translation across a ground plane while making pursuit eye movements to a target moving left and right sinusoidally. They reported that the perceived self-motion path oscillated left and right at the same frequency as the pursuit movement. We call this misperception of the depicted selfmotion the slalom illusion. The illusion manifests a failure to compensate fully for the sinusoidal gaze rotation.

In developing models for the gaze-compensation process in self-motion estimation, it is useful to first consider stimuli in which the retinal motion is due to rotation only. Freeman and Banks (1998) and Freeman (1999) developed a linear model of gaze-rotation compensation for the Filehne illusion. The Filehne illusion occurs when an observer makes a pursuit eye movement while being presented a target that is stationary relative to the head; the target usually appears to move opposite to the eye movement (Filehne, 1922). In order to make the target appear stationary relative to the head - that is, to null its perceived head-centric motion-the target must actually move in the direction of the eye movement. If the nulling motion creates the retinal image motion that is normally produced by a pursuit eye movement, the nulling motion is a simulated eye rotation. In previous studies of the Filehne illusion, the pursuit target velocity was constant during the observation period. Because of this, the percept could be rendered stationary by adjusting the amplitude of the target's velocity (i.e., by adjusting the speed). This can be shown with Equation 1 above. To set the target's motion so that it appears stationary, $\hat{\mathbf{H}}$ must be set to 0 . Then the target velocity yielding a stationary percept is

$$
\mathbf{H}=\mathbf{P}\left[1-\frac{e}{r}\right] .
$$

If $e / r=1$, observers should set the target's head-centric speed to 0 and perceive the target as stationary; gaze compensation is veridical. The standard Filehne illusion paradigm, therefore, allows one to determine the ratio of gains associated with the gaze-compensation process, but not the individual gains themselves.

Gaze compensation may have timing errors as well, and these can be investigated by using sinusoidal eye pursuits. A sinusoidal pursuit (P) and a sinusoidally moving target (H) can be represented by polar vectors with radius representing amplitude and angle representing phase. These vectors are shown schematically in Figure 1. The pursuit and target motion give rise to retinal motion, $\mathbf{R}=\mathbf{H}-\mathbf{P}$, that is also sinusoidal. The conversion from retino-centric to head-centric velocity is given by the following:

$$
\mathbf{H}=\mathbf{R}+\mathbf{P} .
$$

Suppose that the visual system's measurements of $\mathbf{R}$ and $\mathbf{P}$ can be characterized by isotropic, shift-invariant linear operators, $\ell_{r}$ and $\ell_{e}$, respectively. From Equation 3, perceived head-centric motion $(\hat{\mathbf{H}})$ would be as follows:

$$
\hat{\mathbf{H}}=\ell_{r}(\mathbf{H}-\mathbf{P})+\ell_{e}(\mathbf{P})
$$

$\ell_{r}$ and $\ell_{e}$ can introduce amplitude and phase errors. As described by Freeman and Banks (1998) and Freeman 
A) Filehne Illusion, Amplitude Errors Only

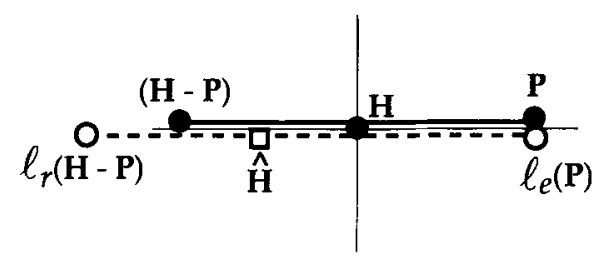

B) Amplitude and Phase Errors

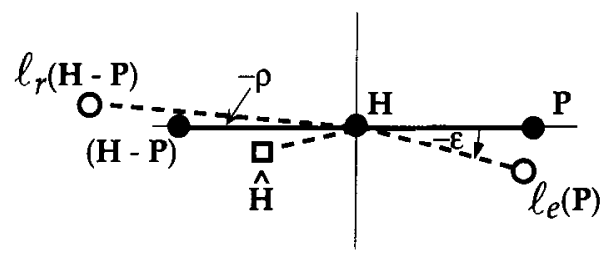

C) Nulling the Filehne Illusion

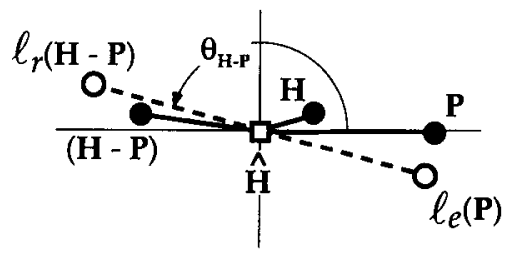

Figure 1. Linear model of amplitude and phase errors that could account for the Filehne illusion during sinusoidal pursuit. Using polar coordinates, amplitude and phase are represented by vector length and direction, respectively. Closed circles represent physical quantities and open circles estimates of those quantities, with open squares defining the resulting motion perceived by the observer. (A) Eye pursuit $(\mathrm{P})$ over a stationary object $(\mathrm{H}=0)$ gives rise to equal and opposite retinal motion $(H-P)$. If no timing errors exist, the Filehne illusion is determined by the relative difference between estimates of pursuit and retinal motion. In the example, pursuit is assumed to be recovered veridically $(e=1$, $\left.\varepsilon=0^{\circ}\right)$. Hence, $P$ and $\ell_{e}(P)$ have equal amplitude and phase. Retinal motion is assumed to be overestimated $\left(r>1, \rho=0^{\circ}\right)$ and so the lengths of $(H-P)$ and $\ell_{r}(H-P)$ differ. The sum of $\ell_{e}(\mathbf{P})$ and $\ell_{r}(\mathbf{H}-\mathbf{P})$, defined as $\hat{\mathbf{H}}$, determines the amplitude of the $\mathrm{Fi}-$ lehne illusion. (B) Introduction of phase errors results in the estimates of extraretinal and retinal motion being rotated away from the horizontal axis, along with their vector sum. Thus, in principle, a stationary object could be perceived to move with incorrect amplitude and phase. (C) To null the Filehne illusion, the observer must introduce motion $(\mathrm{H})$ such that the vector sum of $\ell_{e}(\mathbf{P})$ and $\ell_{r}(\mathbf{H}-\mathbf{P})$ is $\mathbf{0}$. Assuming linearity, one can determine the gain ratio $(e / r)$ and phase difference $(\varepsilon-\rho)$ between extraretinal and retinal signals. The diagram shows why the gains and phases cannot be determined individually: The open circles are constrained to lie at equal distances on opposite sides of the origin, but as a pair they can be arbitrarily rotated or moved radially and still yield the same gain ratio and phase difference.

(1999), amplitude errors are characterized by two gains: retinal gain $r$ and extraretinal gain $e$. Phase errors are here characterized by two phase terms: retinal phase $\rho$ and extraretinal phase $\varepsilon$. As described below, we can estimate the ratio of $e$ and $r$ and the difference between $\rho$ and $\varepsilon$ by hav- ing the observer adjust the amplitude and phase of $\mathbf{H}$ until the display appears stationary with respect to the head.

When the target display is actually stationary $(\mathbf{H}=0)$, its perceived head-centric motion $\hat{\mathbf{H}}$ is usually opposite to the direction of the eye pursuit. Figure 1A shows how this might occur if the Filehne illusion were based on amplitude errors alone. The closed circles represent physical speeds and the open circles possible estimates of those speeds. In the example, the extraretinal signal veridically estimates the pursuit $(e=1)$ while the retinal signal over-estimates retinal motion $(r>1)$. This gives a Filehne illusion indicated by the open square. During sinusoidal pursuit (Figure 1B), both amplitude and phase errors can produce distortions. In this polar representation, amplitude is equal to the length of each vector while phase is equal to its direction. The gains are the same as in Figure $1 \mathrm{~A}$, but this time $\ell_{r}$ and $\ell_{e}$ both lag their respective inputs by $-\rho$ and $-\varepsilon$. The Filehne illusion is the vector sum of $\ell_{r}(\mathbf{H}-\mathbf{P})$ and $\ell_{e}(\mathbf{P})$, which is represented by the open square.

To make the stimulus appear stationary relative to the head, the observer sets $\hat{\mathbf{H}}=0$. To do so, he/she must add motion to $\mathbf{H}$ with a phase similar to $\mathbf{P}$. When the appropriate amplitude and phase of motion have been added, we have from Equation 4:

$$
\ell_{r}(\mathbf{H}-\mathbf{P})=-\ell_{e}(\mathbf{P}) .
$$

This situation is represented by the open circles in Figure $1 \mathrm{C}$; we refer to this state as the null point. Once the percept is nulled, there are two independent equations, one for the gains and one for the phases. The ratio of extraretinal to retinal gain is as follows:

$$
\frac{e}{r}=\frac{|\mathbf{H}-\mathbf{P}|}{|\mathbf{P}|}
$$

The difference in extraretinal and retinal phases is as follows:

$$
\varepsilon-\rho=\theta_{\mathbf{H}-\mathbf{P}}-\theta_{\mathbf{P}}+\pi,
$$

where $\theta_{\mathbf{H}-\mathbf{P}}$ is the phase of the retinal motion at the null point and $\theta_{\mathbf{P}}$ is the phase of the pursuit. To reiterate, it is important to note that the gains and phases of the extraretinal and retinal processes cannot be determined individually with this technique (see also Freeman \& Banks, 1998).

We can now extend this analysis to gaze compensation in self-motion perception. The primary difference from the analysis above is that a translational component is added to the display (depicting forward motion). From standard flow equations (Bruss \& Horn, 1983; LonguetHiggins \& Prazdny, 1980), the retinal flow (F) at point $[x, y]$ in the image plane is the sum of translational $\left(\mathbf{F}_{\mathbf{T}}\right)$ and rotational $\left(F_{R}\right)$ components: $F=F_{T}+F_{R}$. From the analysis above, we characterize the visual system's measurement of retinal flow $(\hat{\mathbf{F}})$ with a linear operator $\left(\ell_{r}\right)$. From the superposition principle,

$$
\hat{\mathbf{F}}=\ell_{r}\left(\mathbf{F}_{\mathbf{T}}\right)+\ell_{r}\left(\mathbf{F}_{\mathbf{R}}\right)
$$


The rotational flow $\mathbf{F}_{\mathbf{P}}$ due to a horizontal eye movement $\mathbf{P}$, is $\mathbf{F}_{\mathbf{P}}=-\mathbf{k P}$, where

$$
\mathbf{k}=\left[\begin{array}{c}
x^{2}+1 \\
x y
\end{array}\right]
$$

Thus:

$$
\hat{\mathbf{F}}_{\mathbf{P}}=-\mathbf{k} \ell_{e}(\mathbf{P})
$$

where $\ell_{e}$ is the linear operator estimating the eye pursuit. Subtracting $\hat{\mathbf{F}}_{\mathbf{p}}$ from $\hat{\mathbf{F}}$ yields an estimate of $\mathbf{F}_{\mathbf{T}}$, from which the direction of self-motion can be determined.

As with the Filehne illusion, the gaze-compensation process is not completely veridical, so when observers look at a stimulus depicting linear translation across a ground plane while they make sinusoidal pursuits left and right, they perceive a sinusoidal oscillation in their path: They experience the slalom illusion. In the experiments reported below, we measured the amplitude and phase errors in gaze compensation by having observers add a rotation $(\mathbf{H})$ to the display until the path appeared straight. $\mathbf{H}$ and $\mathbf{P}$ were constrained to rotate about the same vertical axis, so the rotational flow in the retinal image became

$$
\mathbf{F}_{\mathbf{R}}=-\mathbf{k}(\mathbf{P}-\mathbf{H}) \text {. }
$$

Observers adjusted the amplitude and phase of the rotation $\mathbf{H}$. We assume that the path appears straight when $\ell_{r}(\mathbf{H}-\mathbf{P})=-\ell_{e}(\mathbf{P})$.

Following the analysis of the Filehne illusion represented by Equations 1-7, we can derive similar equations for the gain ratio and phase difference of the gaze-compensation process in path perception. By comparing the values obtained from the Filehne experiments and the self-motion experiments, we can then determine the degree to which gaze compensation is similar in the two tasks.

\section{GENERAL METHOD}

Six observers, all with corrected visual acuities of $20 / 20$ or better, participated. Two (T.C.A.F. and M.S.B.) had considerable experience as psychophysical observers and were aware of the experimental hypotheses. Two (S.M.E. and B.T.B.) had considerable experience, but were unaware of the hypotheses. The other two observers (S.J.M.F. and G.D.H.) had little experience and were unaware of the hypotheses.

The stimuli were random-dot ground planes generated on a Power Macintosh 9500/132 and displayed with an Electrohome ECP-4000 projector on a $97 \times 78.5 \mathrm{~cm}$ rear-projection screen. Background luminance was $\sim 0.01 \mathrm{~cd} / \mathrm{m}^{2}$ and dot luminance was $\sim 11 \mathrm{~cd} / \mathrm{m}^{2}$. The length of the ground plane was truncated at $48 \mathrm{~m}$ from the observer. The width of the ground plane was $114.29 \mathrm{~m}$ and 297 dots were placed pseudo-randomly within this area. The simulated eye height was $1.6 \mathrm{~m}$. For motion sequences depicting forward translation, dots moving behind the observer were wrapped back to the rear edge of the ground plane so that it remained $48 \mathrm{~m}$ from the observer throughout the motion sequence. A rectangular software window clipped the stimuli so that they subtended $100^{\circ} \times 33^{\circ}$ from the $42-\mathrm{cm}$ viewing distance used. The dots themselves were composed of $2 \times 2$ clusters of pixels, each pixel subtending approximately $9.8^{\prime} \times 9.8^{\prime}$ of arc. Using an anti-aliasing routine (Georgeson, Freeman, \& Scott-Samuel, 1996), the centroid of each $2 \times 2$ cluster could be moved smoothly. Dot positions were updated at the
$75-\mathrm{Hz}$ frame rate of the projector. The combination of fast refresh rate and anti-aliasing yielded an appearance of smooth dot motion. ${ }^{1}$

For the issues under examination in this paper, it is important that environmental features, such as the edge of the display screen, be made invisible. To accomplish this, the room was made completely dark except for the display. With this setup, no environmental features could be seen during the stimulus presentatio $\prod n s$.

The observer's head was generally held stationary in a standard head- and chinrest. Viewing was monocular. Observers were instructed to maintain fixation on a small target $1.9^{\circ}$ above the clipped horizon of the ground plane. During stimulus presentations, the fixation target moved sinusoidally and horizontally; it underwent one period of sinusoidal motion before presentation of the ground plane in order to facilitate accurate eye pursuit.

\section{Experiment 1}

\section{Gaze Compensation and the Filehne Illusion}

We first examined gaze compensation for the Filehne illusion by setting the simulated forward translation to zero.

\section{Method}

Observers viewed the ground plane while making horizontal eye pursuits. The simulated translation was zero. A sinusoidal simulated rotation $\mathbf{H}$ was added to the ground plane with random initial amplitude and phase, but with the same frequency as the pursuit target. The rotation of the ground plane was about a vertical axis passing through the observer's eye; this is the same as the simulated eye rotations in previous research on heading perception (e.g., Royden et al., 1992; Royden et al., 1994; Warren \& Hannon, 1988, 1990). Observers adjusted the amplitude and phase of $\mathbf{H}$ until the ground plane appeared stationary with respect to the head.

On each stimulus presentation, the fixation target appeared first and moved left and right sinusoidally. One pursuit period later, the ground plane appeared and was displayed for two more periods. After each presentation, observers adjusted the amplitude or phase of the simulated rotation in an attempt to make the ground plane appear stationary relative to the head. Phase was adjusted first in steps of $40^{\circ}, 10^{\circ}$, or $2.5^{\circ}$. Once an adjustment was made, a new display appeared with the new phase. The procedure was repeated until the observer had minimized the apparent head-centric motion of the ground plane. Amplitude was then adjusted in steps of $1 / 2,1 / 4$, or $1 / 8$ octave until the apparent motion was again minimized. Observers adjusted phase and amplitude, switching from one to the other, until the ground plane appeared stationary relative to the head.

All combinations of two pursuit frequencies $(1 / 3$ and $2 / 3 \mathrm{~Hz})$ and five amplitudes $\left(5^{\circ}-15^{\circ} \text { in } 2.5^{\circ} \text { steps }\right)^{2}$ were presented three times, giving a total of 30 adjustments. Each experimental session investigated 1 of the 10 possible conditions. Conditions were presented in randomized blocks. Four or more practice sessions were completed before any data were collected.

\section{Results and Conclusions}

If the gains and phases in Equations 6 and 7 are fixed, observers' amplitude and phase settings should be the same for different pursuit amplitudes and frequencies. We tested this by presenting a range of amplitudes and frequencies. ${ }^{3}$

The results are displayed in Figures 2 and 3. Figure 2 represents the settings $(\mathbf{H})$ in polar format; the left and right panels show the settings for temporal frequencies of $1 / 3$ and $2 / 3 \mathrm{~Hz}$, respectively, and the upper and lower rows show settings for different observers. The amplitudes of the settings have been normalized, so the polar coordinate $[1,0]$ corresponds with a setting equal in phase 


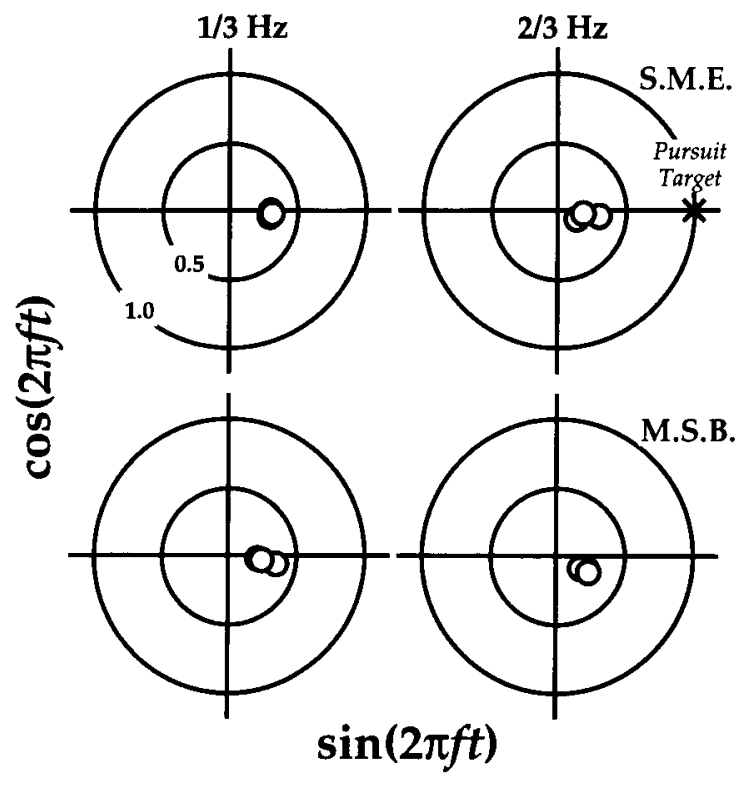

Figure 2. Amplitude and phase of simulated eye rotation required to null the Filehne illusion during sinusoidal pursuit. Each row corresponds to data for a different observer. Two pursuit target frequencies, $1 / 3$ and $2 / 3 \mathrm{~Hz}$, are shown on the left and right, respectively. All amplitudes are normalized to the pursuit target amplitude (the cross in the upper right panel) such that the outer circle has a radius of 1 and the inner circle a radius of 0.5 .

and amplitude to the pursuit target velocity. The target velocity is represented by the $X$ in the upper right panel. We assume that the pursuit eye movements had gains of $\sim 1.0$ and phases of $\sim 0$ with respect to the pursuit target; pursuit acçuracy was verified in Experiment 2A (see Figure 4).

If observers compensated completely for gaze rotations, they would set $\mathbf{H}$ to $[0,0]$. The phases of the $\mathbf{H}$ settings were close to $0(M=-11.24, S D=9.95)$, but the amplitudes were not $(M=0.26, S D=0.06)$. Thus, observers put a rotation into the display in the direction of the pursuit eye movement in order to produce the percept of stationarity relative to the head. This behavior is consistent with prior estimates of the Filehne illusion.

We can estimate the phase differences and gain ratios by applying Equations 6 and 7 to the data of Figure 2 . Those differences and ratios are displayed in Figure 3 as a function of the pursuit target amplitude. The phase differences, $\varepsilon-\rho$, for the $\mathbf{H}$ settings are shown in the upper panel and the gain ratios, $e / r$, are shown in the lower panel. The filled and unfilled symbols represent data when the pursuit frequency was $1 / 3$ and $2 / 3 \mathrm{~Hz}$, respectively. The gain ratios range from 0.65 to 0.85 , independent of experimental condition; this suggests that the amplitude of gaze compensation is lower than is required for veridical compensation. The phase differences are nearly 0 (although very slightly positive) for all conditions, which suggests that the gaze-compensation process has no significant timing error. Therefore, the Filehne illusion during sinusoidal eye movements is caused by a mismatch between the amplitudes of retinal and extraretinal motion estimates and not by a timing error between retinal and extraretinal estimates.

\section{Experiment 2 \\ Amplitude and Phase Errors Accompanying Path Perception}

If the gaze compensation examined in Experiment 1 is a general process that is involved in self-motion estimation as well, we should observe similar findings in a selfmotion task. Experiment $2 \mathrm{~B}$ examined this by using a selfmotion display. Specifically, we added observer translation to the display and asked observers to adjust the ground-plane rotation $(\mathbf{H})$ until the self-motion path appeared straight. In order to determine the characteristics of the gaze-compensation process per se, one needs to know the amplitude and phase of pursuit eye movements for the conditions of the experiment. Thus, in Experiment 2A we measured pursuit amplitude and phase when observers were viewing selected stimuli from Experiment 2B. We first describe the pursuit measurements and then the psychophysical data.

\section{Method}

Eye movements were recorded for a variety of experimental conditions: (1) pursuit with no visible background, (2) pursuit in the presence of simulated forward translation, and (3) pursuit in the presence of translation and simulated gaze rotation. The rotation in the third condition had a phase and amplitude determined by the ob-
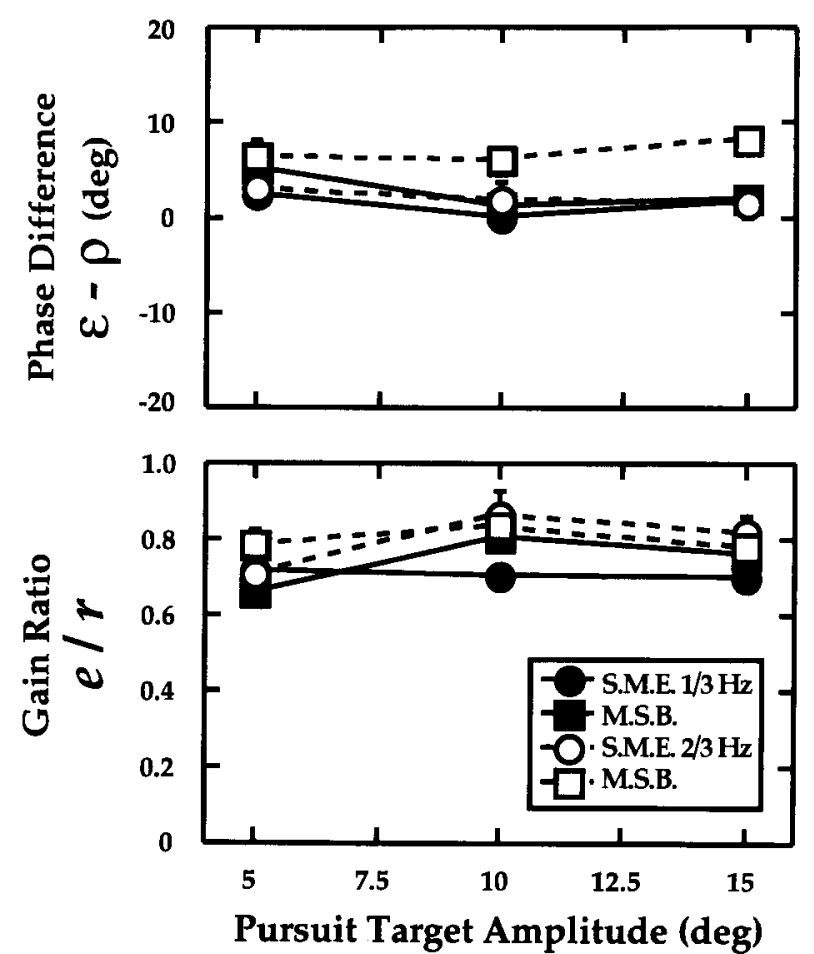

Figure 3. Estimates of phase difference (upper panel) and gain ratio (lower panel) obtained by applying Equations 6 and 7 to the data of Figure 2. Pursuit was assumed to be veridical. Closed symbols correspond to the $1 / 3-\mathrm{Hz}$ condition and open symbols to the $2 / 3-\mathrm{Hz}$ condition. Error bars are $\pm 0.5 S D$. 
server's settings in Experiment 2B, so that we could use the measurements to estimate pursuit accuracy in that experiment at the null point. Observers could conceivably have ignored the ground plane in order to improve pursuit accuracy, so we minimized this possibility by asking them to indicate which of the two ground-plane displays contained the straighter path. This decision was made following a randomly ordered triplet of trials consisting of one example of the three conditions described above.

Eye movements were measured using a head-mounted, infrared limbus eye tracker (Eye Trac Model 210, Applied Sciences Laboratories). Eye movement records were sampled at $100 \mathrm{~Hz}$. The optical frame of the tracker restricted the central field of view to approximately $40^{\circ} \times 24^{\circ}$; some of the display was also visible outside this frame. At the beginning of each session, the eye tracker was calibrated using the following procedure. Observers fixated 9 dots in succession at different horizontal positions. Eye tracker output was then compared with the known dot positions in order to calibrate for bias and gain.

During the experiment itself, the fixation target moved left and right sinusoidally. The eye movement record was stored for the last two periods of each pursuit and was then analyzed off line in MATLAB. Saccades were not removed. A fast Fourier transform was used to determine amplitude and phase spectra. Almost all the power was concentrated at the fundamental frequency of the pursuit target, so we summarized performance with the amplitude and phase at that frequency. ${ }^{4}$

\section{Results and Conclusions}

Figure 4 plots in polar coordinates the mean amplitudes and phases of the pursuit movements. The left and right panels show the results for $1 / 3$ and $2 / 3 \mathrm{~Hz}$, respectively. The phase and amplitude of the pursuit target are represented by the $\mathrm{X}$ in the right panel. Pursuit target amplitudes have been normalized to 1 and the response amplitudes have, therefore, been divided by the same normalization factors. The ratio of response amplitude divided by target amplitude is defined as the pursuit gain. Each data point, therefore, represents pursuit gain and phase for a given condition averaged across the 3 observers, with different symbols representing different conditions. Pursuit gains in all conditions were very close to 1.0 . There was a small phase lag that did not vary systematically with viewing condition. Thus, for the conditions of Experiment 2, pursuit eye movements were quite accurate.

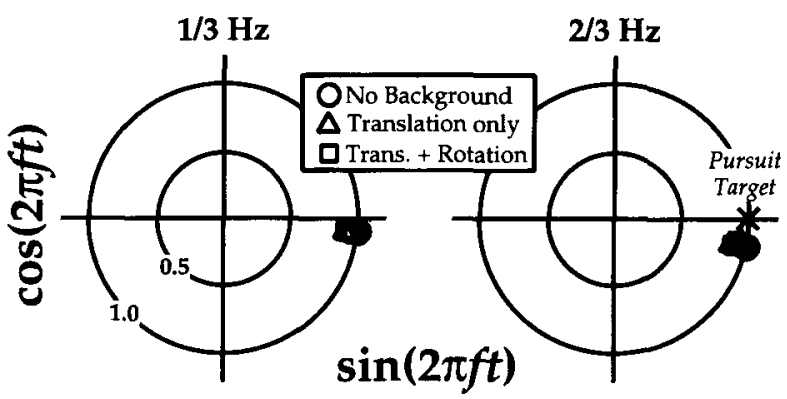

Figure 4. Measured amplitude and phase of eye movements for 1/3- and $2 / 3-\mathrm{Hz}$ conditions (left and right, respectively). Each symbol is the mean across observers at one of the five amplitudes studied; all amplitudes have been normalized to the pursuit target amplitude as in Figure 2. Different symbol types denote the three stimulation conditions described in the text.
Observers were asked on each trial to indicate the stimulus that yielded a straighter perceived path. Condition 3 was chosen $91 \%$ of the time with $1 / 3-\mathrm{Hz}$ pursuit and $82 \%$ with $2 / 3-\mathrm{Hz}$ pursuit. The fact that they were significantly above chance shows that observers attended to the stimulus during eye-movement recording.

Having established that pursuit eye movements were quite accurate when observers viewed (and made judgments concerning) self-motion displays, we now describe the main part of Experiment 2: While making sinusoidal pursuits, observers adjusted the amplitude and phase of simulated gaze rotation until their perceived self-motion path was straight.

\section{Method}

The method was identical to that of Experiment 1 except that the ground plane also simulated observer translation along a straight path toward the center of the screen. Depicted translation speed was 10 eye heights $/ \mathrm{sec}$, which is equivalent to $16 \mathrm{~m} / \mathrm{sec}$ at the simulated eye height of $1.6 \mathrm{~m}$. This display, coupled with a sinusoidal eye pursuit, produces a compelling illusion of slaloming across the ground plane. Observers adjusted the amplitude and phase of the simulated gaze rotation until their perceived self-motion path was straight.

\section{Results and Conclusions}

Figure 5 shows the results in polar coordinates. The left and right columns show data for temporal frequencies of $1 / 3$ and $2 / 3 \mathrm{~Hz}$, respectively, and the rows represent data from different observers. The squares represent the amplitude and phase of the observers' pursuits (P; obtained in eye movement recordings described above) and the circles represent the amplitude and phase of their psychophysical settings $(\mathbf{H})$. As before, the amplitudes have been normalized, so a value of 1.0 represents data for which the setting or response amplitude was equal to the pursuit target amplitude. Each symbol represents data from a different pursuit target amplitude.

If gaze compensation were veridical, observers would perceive a linear path when the simulated gaze rotation $\mathbf{H}$ was $[0,0]$. In fact, $\mathbf{H}$ was typically set to half the amplitude of and in roughly the same phase as the eye pursuit. Thus, as in Experiment 1, gaze compensation was not veridical; observers again required a simulated rotation in the direction of the eye pursuit before the depicted self-motion path appeared straight.

We can again use Equations 6 and 7 to calculate phase differences and gain ratios for the gaze-compensation process. Figure 6 shows the estimated phase differences and gain ratios in the upper and lower panels, respectively. The open symbols in Figure 6 represent the phases and gains under the assumption of accurate pursuit and the filled symbols the phases and gains when the actual eye pursuits are included in the analysis. The inclusion of the actual pursuits has a small effect on the phase differences and no systematic effect on the gain ratios; the phase effect is due to the small lag of eye pursuits relative to the pursuit target.

The results are in many respects similar to the findings for the Filehne illusion. As with the Filehne illusion data, the gain ratio was significantly greater than 0 and the 


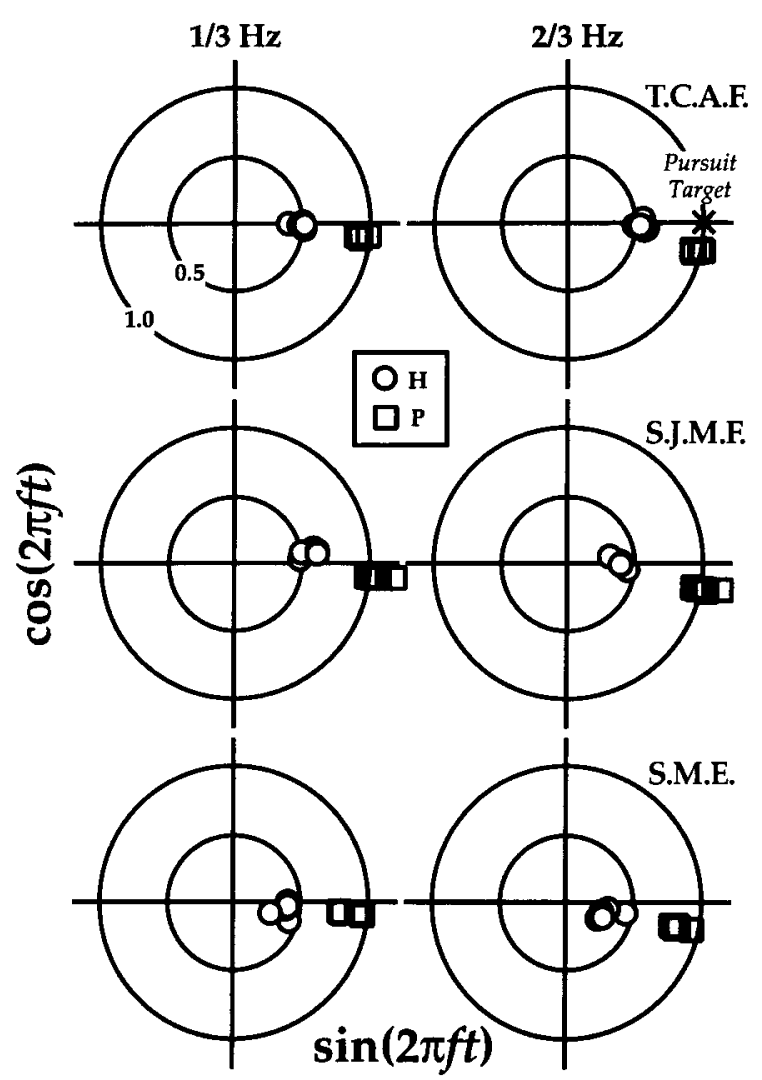

Figure 5. The amplitude and phase of the simulated eye rotation that nulled the slalom illusion (circles) together with the measured eye pursuit (squares) found for each observer in Experiment $2 \mathrm{~A}$. All amplitudes have been normalized to the pursuit target amplitude (the cross in the upper left panel). Left and right columns give data for the $1 / 3-$ and $2 / 3-\mathrm{Hz}$ conditions, respectively. Each row shows the data for 1 observer.

phase difference was small, which means that the slalom illusion is caused by a mismatch between the amplitudes of retinal and extraretinal motion estimates and not by a timing error between the retinal and extraretinal processes. In addition, there was no systematic change in phase difference or gain ratio as a function of pursuit target amplitude; this is consistent with the model presented above.

In one respect, the results are dissimilar from our findings on the Filehne illusion: The gain ratio was systematically lower for the slalom illusion than for the Filehne illusion. A lower gain ratio with self-motion displays is inconsistent with the hypothesis that part of the gaze|compensation process in self-motion perception is the result of purely retinal compensation. This type of " $\mathrm{mix}$ ture model" (Banks et al., 1996) should, if anything, increase the gain ratio relative to the Filehne condition, assuming that the latter arises from extraretinal gaze compensation alone (see General Discussion). We hasten to point out, however, that different observers participated in the two experiments, so one cannot determine whether the dissimilarity in results is due to differences in the information content of the stimuli or in the observers. ${ }^{5} \mathrm{Be}$ - cause of this ambiguity, we examined the effect of translation speed on gain ratio in the next experiment.

\section{Experiment 3 \\ Effect of Forward Translation Speed on Gain Ratio}

If the gaze-compensation process is general and applies equally to the Filehne illusion condition (Experiment 1 ) and the slalom illusion condition (Experiment 2), the gain ratio should be the same whether the depicted translation speed is zero or not. To test this, we next measured gain ratios for a wide range of translation speeds, including the Filehne condition, where the speed was zero.

\section{Method}

The stimuli and procedure were the same as in Experiment 2 with the following exceptions. The translation speed was varied from 0 to 12.5 eye heights $/ \mathrm{sec}$. The pursuit target was presented at only one frequency and one amplitude: $1 / 3 \mathrm{~Hz}$ and $10^{\circ}$. Two pursuit periods were displayed and the ground plane was visible during the second period. Each condition was presented five times. To simplify the observer's task, the phase of the simulated gaze rotation was fixed at $0^{\circ}$ and observers adjusted rotation amplitude. Initial amplitude was random and step size was $\pm 10^{\circ}, \pm 2.5^{\circ}$, or $\pm 0.625^{\circ}$. Eye movements were not recorded.

There were two unwanted cues in Experiment 1 that could have been used to aid performance. The first was the appearance and disappearance of dots at the edges of the display screen. When the sim-

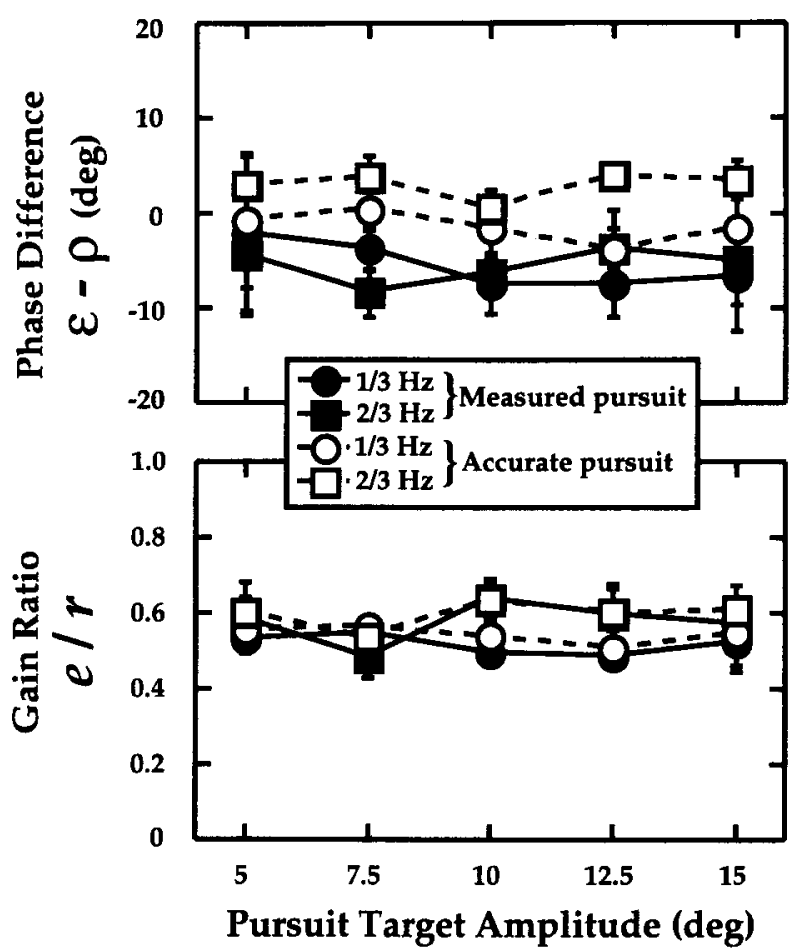

Figure 6. Estimates of phase difference (upper panel) and gain ratio (lower panel) obtained by applying Equations 6 and 7 to the data of Figure 5. Each point is the mean across the 3 observers, with error bars denoting $\pm 0.5 S D$. Open symbols represent the estimates assuming veridical pursuit. Closed symbols represent the estimates obtained using the measured pursuits of Figure 5. Squares correspond to the $1 / 3-\mathrm{Hz}$ condition and circles to the $2 / 3-$ $H z$ condition. Error bars are $\pm 0.5 S D$. 
ulated rotation is nonzero, dots appear and disappear at the edges. Thus, observers could have set the rotation to zero by making adjustments until no dots appeared or disappeared. The second cue was a just-discernible flicker of the dots when they were moving slowly across the screen. Again, observers could have used this cue by adjusting the rotation until the dots did not flicker. None of the observers in Experiment 1 noticed these cues; nevertheless, we removed them in Experiment 3 . The first was removed by using a moving clipping window with a width of $75^{\circ}$. The window moved with the pursuit target so that dot appearance and disappearance occurred for nearly all values of simulated rotation including the veridical value. The second cue was made uninformative by randomly perturbing dot positions by \pm 0.2 pixels ( $\pm 4 \mathrm{~min}$ arc) on each frame.

\section{Results and Conclusions}

Gain ratios were calculated from the observers' settings by using Equation 6 ; eye pursuit was assumed to be accurate. The results are displayed in Figure 7, which plots gain ratio, $e / r$, as a function of the depicted translation speed. Different symbols represent data from different observers.

Veridical gaze compensation would yield gain ratios of 1.0. Notice that the observed ratios were generally less than 1.0 , which shows that gaze compensation is nonveridical for translation speeds varying from 0 to 12.5 eye heights/sec. There is no clear correlation between gain ratio and translation speed. The results are consistent with the hypothesis that a similar, extraretinal compensation process occurs at all translations speeds, including a speed of zero.

\section{GENERAL DISCUSSION}

\section{Interpreting Amplitude and Phase Errors}

Our results show that two illusions of head-centric motion-the Filehne and slalom illusions - arise principally from amplitude errors in the gaze-compensation process. Phase errors are small and not a significant factor.

The amplitude errors arise from a mismatch in the gains of the extraretinal and retinal components of gaze compensation. The measure of amplitude errors was the gain

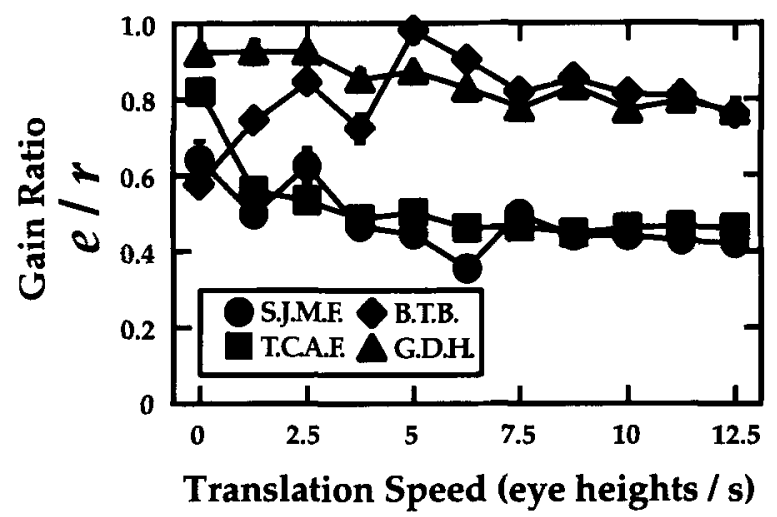

Figure 7. Gain ratio as a function of translation speed assuming veridical pursuit. The pursuit target moved at $1 / 3 \mathrm{~Hz}$ with an amplitude of $10^{\circ}$. Each symbol corresponds to a different observer. Error bars are $\pm 0.5 S D$. ratio (see Equation 6), and that ratio was typically less than 1. As Freeman (1999) and Freeman and Banks (1998) have pointed out, a gain ratio less than 1 does not imply that extraretinal signals underestimate eye pursuit amplitude (Mack \& Herman, 1973, 1978; Wertheim, 1987; Yasui \& Young, 1975); it simply means that the gain of the extraretinal signal is less than the gain of the retinal signal for our experimental conditions. Both signals could, in principle, be overestimating the amplitude of their respective inputs.

Because the phase errors were small, any mismatch in timing between extraretinal and retinal components must be small. Again, this does not mean that the phases of the extraretinal and retinal components of gaze compensation are zero with respect to the pursuit eye movement; it means that the phases of the two components are similar to each other. They probably both lag their respective inputs by roughly equal amounts, but our technique does not allow such a determination.

\section{Similarity Between \\ Filehne Illusion and Slalom Illusion}

We compared gaze compensation in the Filehne and slalom conditions in an attempt to determine how much of the compensation in self-motion judgments is accomplished via extraretinal signals. Our reasoning was that retinal compensation is in principle possible in self-motion tasks (see, e.g., Longuet-Higgins \& Prazdny, 1980), but would be unavailable during the Filehne task. It is conceivable, however, that gaze compensation in the Filehne condition could be subserved by retinal-image-based gaze compensation. Specifically, one could in principle judge the stationarity of our displays without the use of extraretinal signals by assuming that large rigid backgrounds are stationary relative to the head (Wallach, 1959; see Wertheim, 1994, for review). However, this strategy would not work in our experiments: If one assumed that large backgrounds are stationary, our stimuli should have appeared stationary at all amplitudes (and phases) of the simulated eye rotation added to the display. This is clearly not the case because observers required specific amplitudes and phases of simulated rotation to make the ground plane appear stationary. Thus, we should be safe in assuming that extraretinal compensation is required in the Filehne condition.

The results of Experiment 3 show that gain ratio decreases slightly as translation speed increases from 0 (Figure 7). We presume that only extraretinal compensation occurs when translation speed is zero. Thus, if extraretinal and retinal compensation both operated during selfmotion judgments (the mixture model), one would expect the gain ratio to be higher when the translation speed is nonzero. If anything, the gain ratio decreased slightly as the speed was increased from zero. Thus, we have no evidence that retinal-based gaze compensation occurred in the self-motion task. Warren and Li (1998) argued that greater retinal-based compensation occurs when the ground 
plane has denser textures than the random dots used here. Thus, it remains possible that we would have observed more evidence for retinal-based compensation had we used such textures.

\section{Relationship to Previous Literature}

The existence of the slalom illusion implies that the perception of direction of self-motion is not always accurate. Previous studies of perceived self-motion direction have shown small, but consistent, errors when a straight-path translation is presented during a constant eye pursuit (Banks et al., 1996; Crowell et al., 1998; Ehrlich et al., 1998; Royden et al., 1992; Royden et al., 1994; van den Berg \& Brenner, 1994). It is not clear whether the model and gain ratios reported here are quantitatively consistent with those previous observations. Freeman (1999) measured gain ratios in the Filehne and self-motion conditions during constant eye movements. Three observers who participated in that study also participated in the present study: They showed similar gain ratios (for both the Filehne and self-motion conditions) with constant-velocity pursuits and sinusoidal pursuits. The similarity of observed gain ratios implies that the slalom illusion is a manifestation of the same mismatch in extraretinal and retinal speed estimation that occurs during a constant eye movement. Freeman also showed that the small heading errors that occur during real eye pursuit could be nulled with appropriate simulated eye rotation in a pointing task. However, further work will be required to determine whether these observations can quantitatively predict the magnitude of heading error previously reported.

\section{Does a Linear Model With Fixed Gains Suffice?}

The model presented here assumes that the gazecompensation process can be represented by two linear systems, one extraretinally based and the other retinally based. In its current form (e.g., Equation 1), it also assumes that the gain of the extraretinal component is fixed and that the gain of the retinal component varies only with the spatial properties $(\Omega)$ of the visual stimulus. Our results are consistent with these assumptions: Gain ratio and phase difference were mostly unaffected by changes in pursuit frequency and amplitude. We did not, however, present wide ranges of pursuit frequencies and amplitudes, so our experiments did not put the assumptions to a strong test. Further work will be needed to investigate the extent to which the linear model and its assumptions can provide a good account of motion perception during eye movements.

\section{Conclusions}

The Filehne illusion is the misperception of the headcentric speed of a rigid pattern during eye pursuit. We compared this illusion to the novel slalom illusion, in which the perceived direction of self-motion oscillates from side to side during sinusoidal pursuit across a simulated self-motion display. Both illusions may be caused by errors in extraretinal and retinal speed and phase estimation.
By applying a linear model to motion-nulling data, we found that both illusions can be explained by equivalent errors in speed and phase estimation. Our data provide further evidence for the use of extraretinal signals in the perception of self-motion direction during smooth eye pursuit.

\section{REFERENCES}

Banks, M. S., Ehrlich, S. M., Backus, B. T., \& Crowell, J. A. (1996). Estimated heading during real and simulated eye movements. Vision Research, 36, 431-443.

Beintema, J. A., \& VAN den Berg, A. V. (1998). Heading detection using motion templates and eye velocity gain fields. Vision Research, 38, 2155-2179.

Bruss, A. R., \& Horn, B. K. P. (1983). Passive navigation. Computer Vision, Graphics, \& Image Processing, 21, 3-20.

Crowell, J. A., Banks, M. S., Shenoy, K. V., \& Andersen, R. A. (1998). Self-motion perception during head turns. Nature Neuroscience, 1, 732-737.

Ehrlich, S. M., Beck, D. M., Crowell, J. A., Freeman, T. C. A., \& BANKS, M. S. (1998). Depth information and perceived self-motion during simulated gaze rotations. Vision Research, 38, 3129-3146.

FiLEHNE, W. (1922). Über das optische Wahrnehmen von Bewegungen [On optical perception of movements]. Zeitschrift für Sinnephysiologie, 53, 134-145.

Freeman, T. C. A. (1999). Path perception and Filehne illusion compared: Model and data. Vision Research, 39, 2659-2667.

Freeman, T. C. A., \& Banks, M. S. (1998). Perceived head-centric speed is affected by both extraretinal and retinal errors. Vision Research, 38, $941-945$

Freeman, T. C. A., Crowell, J. A., \& Banks, M. S. (1996). Perceived heading during real eye pursuit eye movements is not always accurate [Abstract]. Investigative Ophthalmology \& Visual Science, 37, \$454.

Georgeson, M. A., Freeman, T. C. A., \& Scott-Samuel. N. E. (1996). Sub-pixel accuracy: Psychophysical validation of an algorithm for the fine positioning and movement of dots on visual displays. Vision Research, 36, 605-612.

Gibson, J. J., Olum, P., \& Rosenblatt, F. (1955). Parallax and perspective during aircraft landings. American Journal of Psychology, 68, 372-385.

HeEger, D. J., \& JEPSON, A. D. (1992). Subspace methods for recovering rigid motion I: Algorithm and implementation. International Journal of Computer Vision, 7, 95-117.

Longuet-Higgins, H. C., \& Prazdny, K. (1980). The interpretation of a moving retinal image. Proceedings of the Roval Society of London: Series $B, \mathbf{2 0 8 , 3 8 5 - 3 9 7 . ~}$

Mack, A., \& Herman, E. (1973). Position constancy during pursuit eye movement: An investigation of the Filehne illusion. Quarterly Journal of Experimental Psychology, 25, 71-84.

Mack, A., \& Herman, E. (1978). The loss of position constancy during pursuit eye movements. Vision Research, 18, 55-62.

Perrone, J. A., \& STone, L. S. (1994). A model of self-motion estimation within primate extrastriate visual cortex. Vision Research, 34, 2917-2938

Royden, C. S., BANKs, M. S., \& Crowell, J. A. (1992). The perception of heading during eye movements. Nature, 360, 583-585.

Royden, C. S., Crowell, J. A., \& Banks, M. S. (1994). Estimating heading during eye movements. Vision Research, 34, 3197-3214.

Smith, A. T., \& EdGaR, G. K. (1990). The influence of spatial frequency on perceived temporal frequency and perceived speed. Vision Research, 30, 1467-1474.

Thompson, P. (1982). Perceived rate of movement depends on contrast. Vision Research, 22, 377-380.

VAN DEN BERG, A. V. (1996). Judgements of heading. Vision Research, 36, 2337-2350.

VAN DEN BerG, A. V., \& Brenner, E. (1994). Humans combine the optic flow with static depth cues for robust perception of heading. Vision Research, 34, 2153-2167.

Wallach, H. (1959). The perception of motion. Scientific American, 201, 56-60. 
WARREN, W. H., JR., \& HANNON, D. J. (1988). Direction of self-motion is perceived from optical flow. Nature, 336, 162-163.

WARREN, W. H., JR., \& HANNON, D. J. (1990). Eye movements and optical flow. Journal of the Optical Society of America A, 7, 160-169.

Warren, W. H., \& Li, L. (1998). Perception of heading during simulated rotation: Influence of dot density and depth range [Abstract]. Investigative Ophthalmology \& Visual Science, 39, S893.

Watamaniuk, S. N. J., Grzywacz, N. M., \& Yuille, A. L. (1993). Dependence of speed and direction perception on cinematogram dot density. Vision Research, 33, 849-859.

WERTHEIM, A. H. (1987). Retinal and extraretinal information in movement perception: How to invert the Filehne illusion. Perception, 16, 299-308.

WerTHEIM, A. H. (1994). Motion perception during self-motion-The direct versus inferential controversy revisited. Behavioural \& Brain Sciences, 17, 293-311.

Yasui, S., \& Young, L. R. (1975). Perceived visual motion as effective stimulus to pursuit eye movement system. Science, 190, 906-908.

\section{NOTES}

1. Many of the functions used to generate the stimuli and collect responses are posted at the following website: http://john.berkeley.edu/ MatLab_Experiment_Libraries/ExperimentLibraries.html.
2. The root mean square speed for the pursuit target ranged from $7.4^{\circ}$ to $22.21 \%$ sec for the $1 / 3-\mathrm{Hz}$ condition. For the $2 / 3-\mathrm{Hz}$ condition, the range was $14.8^{\circ}$ to $44.42^{\circ} / \mathrm{sec}$.

3. The model makes no prediction about the frequency dependence of gains and phases; it could be, for example, that the gaze-compensation process is lowpass, so that gain is reduced at higher temporal frequencies. For this reason, we tested only two temporal frequencies.

4 . To determine eye speed from this analysis, one can multiply the resulting position amplitude by $2 \pi f$. Note, however, that this multiplicative constant is canceled by division in subsequent analysis because all speeds are normalized to the pursuit target (e.g., Figure 5). Another technique is to low-pass filter the eye position record, compute its temporal derivative, and then recover the speed amplitude and phase at the appropriate frequency. We found negligible differences between these two types of analysis for our eye movement data.

5. Observer S.M.E. actually participated in both experiments. Collapsing across frequency and amplitude, the mean gains for her data (assuming accurate pursuit) were nearly identical: $0.71(S D=0.07)$ for the Filehne illusion and $0.67(S D=0.08)$ for the slalom illusion.

(Manuscript received February 23, 1999; revision accepted for publication May 12, 1999.) 